Debates en Medicina Intensiva

\title{
Utilidad de las maniobras de reclutamiento (PRO)
}

\author{
F. SUÁREZ SIPMANN \\ Servicio de Medicina Intensiva. Fundación Jiménez Díaz-UTE. Madrid. España.
}

En los últimos años las maniobras de reclutamiento (MR) han despertado un interés creciente por su potencial efecto beneficioso en la protección pulmonar y se han ido introduciendo en la práctica clínica de forma progresiva. Numerosos estudios clínicos y experimentales han descrito los beneficios fisiológicos obtenidos tras la reexpansión pulmonar, si bien éstos no son uniformes debido en parte a la gran heterogeneidad de las MR aplicadas y a la falta de criterios que definan sus objetivos. Por ello hasta la fecha ha sido difícil establecer cuál es el papel que el reclutamiento tiene en el manejo ventilatorio de los pacientes con SDRA. La información obtenida de los estudios recientes ha permitido entender mejor los mecanismos por los que actúa el reclutamiento, la interpretación de su respuesta y sus efectos secundarios, lo que ha contribuido a la mejora en su aplicación práctica. Las MR deben aplicarse de forma protocolizada e individualizada, determinando la presión necesaria para obtener la mayor reexpansión pulmonar razonablemente posible en cada paciente. El ajuste de la PEEP tras el reclutamiento es un aspecto esencial sin el cual las MR no tienen utilidad ni posiblemente justificación. Teniendo en cuenta estos aspectos fundamentales, estamos más cerca de, como cree el autor, demostrar el beneficio de las MR en la protección pulmonar y con ello mejorar el pronóstico de los pacientes con SDRA.

PALABRAS CLAVE: Reclutamiento alveolar. PEEP. Colapso. Protección pulmonar. Lesión asociada a ventilación mecánica.

Correspondencia: Dr. F. Suárez Sipmann.

Servicio de Medicina Intensiva. Fundación Jiménez Díaz-UTE.

Avda. de los Reyes Católicos, 2. 28040 Madrid. España.

Correo electrónico: fsuarez@fjd.es

Manuscrito aceptado el 10-10-2008.

\section{UTILITY OF RECRUITMENT MANEUVERS (PRO)}

In recent years lung recruitment maneuvers (RM) have awakened an increasing interest due to their potential beneficial effects in lung protection so that they have been progressively introduced into clinical practice. Many clinical and experimental studies have described the physiological benefits obtained after lung re-expansion although these benefits are not uniform, partly because of the wide heterogeneity of the RMs applied and lack of criteria defining their goal. Therefore, to date it has been difficult to establish the role of recruitment in the ventilatory management of ARDS patients. However, the information obtained from recent studies has improved our understanding regarding the mechanisms governing lung recruitment, interpretation of its response and its side effects and this has strongly contributed to its improved practical application. Lung recruitment must be applied in a protocolized and individualized way, establishing the pressure necessary to obtain the reasonably possible maximum lung re-expansion in each patient. Post RM PEEP adjustment is an essential aspect which, if ignored, renders RM useless and possibly without indication. Taking these essential aspects into account we are getting closer to, as the author believes, finally demonstrating the benefit of RM in lung protection and ARDS patients' outcome.

KEY WORDS: Alveolar recruitment. PEEP. Collapse. Lung protection. Ventilation associated lung injury.

\section{INTRODUCCIÓN}

La prevención de la lesión asociada a la ventilación mecánica (LAVM) se ha convertido en un objetivo prioritario en el manejo de los pacientes con el síndrome de distrés respiratorio agudo (SDRA) ${ }^{1}$. 
Existe una amplia evidencia experimental del efecto deletéreo que sobre el pulmón tiene esta lesión. Los mecanismos por los cuales se produce son por un lado la sobredistensión secundaria a un volumen corriente y/o presión respiratoria excesivos y por otro el colapso-reapertura alveolar cíclicos $(\mathrm{CAC})^{2}$. Si bien la protección pulmonar obtenida con la estricta limitación del volumen corriente y de la presión meseta ha quedado sólidamente demostrada ${ }^{3-5}$, el beneficio derivado de la eliminación del colapso pulmonar mediante maniobras de reclutamiento (MR) y el mantenimiento de la estabilidad alveolar mediante el ajuste de PEEP, el llamado concepto de pulmón abierto $^{6}$, ha resultado una hipótesis más difícil de testar clínicamente y en la actualidad el debate respecto a su utilidad y beneficio continua abierto. En la última década han aparecido numerosos trabajos experimentales y clínicos dirigidos a estudiar los efectos fisiológicos y la seguridad a corto plazo de las MR. Aunque muchos de ellos han mostrado respuestas fisiológicas beneficiosas, principalmente en la oxigenación y en la mecánica respiratoria, éstas han sido heterogéneas y en general de corta duración 7 . En ellos se han propuesto diferentes tipos de MR combinando diferentes modos ventilatorios, presiones inspiratorias, tiempos de aplicación y niveles de PEEP. Sin embargo, no existe en la actualidad un consenso acerca de cuál es la forma más eficaz y segura de aplicar una MR ni, por lo tanto, datos concluyentes respecto a su beneficio. Por otro lado, el conocimiento acumulado en estos estudios ha ayudado a entender mejor los mecanismos en los que se basa, sus objetivos y su potencial papel en la protección pulmonar, lo que ha ido aumentando su aceptación y utilización.

\section{FUNDAMENTO DE LAS MANIOBRAS DE RECLUTAMIENTO EN EL SDRA}

La presencia de colapso alveolar en el SDRA está extensamente documentada ${ }^{8,9}$. Estudios recientes han mostrado que el colapso puede afectar de un 20 a un $40 \%$ del volumen pulmonar total en pacientes con SDRA de distinto origen ${ }^{10-13}$. Además del deterioro en el intercambio gaseoso y la mecánica respiratoria, el colapso favorece y amplifica la LAVM. Por un lado, contribuye a la sobredistensión de las zonas aireadas, menos dañadas, que deben acomodar la totalidad del volumen corriente, y por otro el colapso siempre lleva asociado en mayor o menor medida la existencia de CAC. Existe una amplia evidencia experimental de los efectos lesivos del colapso incluso con el empleo de volúmenes corrientes bajos. Sin embargo, éstos pueden atenuarse mediante la aplicación de niveles de PEEP más altos ${ }^{14,15}$ o revirtiendo el colapso mediante reclutamiento y la aplicación de $\mathrm{PEEP}^{16}$. A nivel clínico, Gattinoni et $\mathrm{al}^{13}$ han mostrado que los pacientes con mayor proporción de tejido pulmonar no aireado en la tomografía computarizada (TC) presentan un mayor deterioro funcional y una mayor mortalidad.

El empleo de volúmenes corrientes bajos puede promover el colapso alveolar, sobre todo cuando el nivel de PEEP utilizado es bajo ${ }^{17-19}$. La mera limitación del volumen corriente además puede no conferir la protección pulmonar buscada, puesto que no logra evitar la sobredistensión inspiratoria ${ }^{20,21}$. En un intento de optimizar la protección pulmonar ofrecida por la limitación del volumen corriente, se ha propuesto la utilización niveles de PEEP más elevados. Sin embargo, tres grandes estudios aleatorizados recientes, dirigidos específicamente a intentar demostrar este efecto protector adicional de la PEEP, no han sido concluyentes $^{22-24}$. A la luz de estos resultados desalentadores, cabe plantearse bien que la PEEP realmente no ofrece ventajas adicionales, lo que esta en contradicción con una amplia evidencia experimental de su efecto protector, bien que el mero incremento de PEEP no sea suficiente ya que no consigue la reexpansión pulmonar buscada, y al persistir el colapso, pueda incluso aumentar el riesgo de sobredistensión $^{25}$.

En este contexto, las MR se han propuesto como una medida adyuvante en la protección pulmonar, dirigida a eliminar el colapso. En un pulmón expandido sin colapso, la distribución de la ventilación es más homogénea y en combinación con un nivel de PEEP adecuado se crean las condiciones ideales para minimizar la sobredistensión y el $\mathrm{CAC}^{26}$.

\section{DEFINICIÓN Y OBJETIVO DE LAS MANIOBRAS DE RECLUTAMIENTO}

El reclutamiento se puede definir como la reexpansión de áreas pulmonares previamente colapsadas mediante un incremento breve y controlado de la presión transpulmonar ${ }^{27}$. Está dirigido a crear y mantener una condición libre de colapso hasta lo razonablemente posible («pulmón abierto») ${ }^{6}$, con el fin último de poder instaurar una estrategia de protección pulmonar general. La disminución del colapso tiene como efecto inmediato la mejora en la oxigenación, lo que se ha utilizado como criterio de respuesta al reclutamiento. Sin embargo, estos criterios han sido bastante heterogéneos en los diferentes estudios clínicos basados en cambios relativos como una mejora en la $\mathrm{PaO}_{2} / \mathrm{FiO}_{2}$ del $20 \%{ }^{28}$, del $50 \%{ }^{29}$ o sobre un valor predeterminado ${ }^{30}$ lo que unido a la utilización de niveles diferentes de $\mathrm{FiO}_{2}$, incluso antes y después de aplicar la MR en un mismo estudio ${ }^{30,31}$, ha hecho difícil la evaluación y la comparación de la efectividad de las MR. Recientemente Borges et al ${ }^{10}$ han validado un criterio basado en la oxigenación, ya sugerido por Lachmann ${ }^{6}$, para definir el reclutamiento máximo en pacientes con SDRA utilizando como referencia la TC. Estos autores mostraron que una relación $\mathrm{PaO}_{2} / \mathrm{FiO}_{2} \geq 350 \mathrm{mmHg}$ utilizando una fracción inspirada de oxígeno de 1 correspondía a una masa de tejido pulmonar colapsado inferior al 5\%. Disponer de un criterio clínico objetivo para evaluar la efectividad de una MR en términos de reducción del colapso pulmonar, y no simplemente de su efecto en la oxigenación, permite analizar y aplicar las MR desde la perspectiva de su objetivo primario: la protección pulmonar. 


\section{MANIOBRAS DE RECLUTAMIENTO: VARIABILIDAD EN LA RESPUESTA}

La respuesta al reclutamiento es variable de un paciente a otro y depende de factores tales como el tipo de lesión (pulmonar o extrapulmonar) ${ }^{31}$, la fase evolutiva (precoz o tardía) ${ }^{28,29}$, la severidad de la lesión, la historia del volumen pulmonar previo, el tipo de MR y el nivel de presión alcanzado ${ }^{10}$. La gama de presiones descrita en los estudios clínicos va desde un mínimo de $40 \mathrm{cmH}_{2} \mathrm{O}$ necesarios para reexpandir pulmones sanos colapsados tras la inducción anestésica ${ }^{32}$ hasta $60 \mathrm{cmH}_{2} \mathrm{O}$ utilizados en pacientes con SDRA $^{10,12,33}$.

Es fácil entender que una misma MR empleando un único nivel de presión tendrá una respuesta variable en distintos pacientes, y que para conseguir un reclutamiento efectivo tal y como se definió previamente, cada paciente necesitará un nivel de presión individualizado. Así, el empleo de un único nivel de presión podría resultar en una MR subóptima al no conseguir una reexpansión efectiva si éste fuese demasiado bajo ${ }^{13} \mathrm{o}$ en riesgos innecesarios para el paciente si fuese demasiado elevado ${ }^{33}$. La importancia de la individualización de la maniobra de reclutamiento fue puesta de manifiesto por Borges et al. Utilizaron una MR secuencial en presión controlada partiendo de una PEEP de $25 \mathrm{cmH}_{2} \mathrm{O}$ y de una presión inspiratoria sobre PEEP de $15 \mathrm{cmH}_{2} \mathrm{O}$. Mediante incrementos de PEEP en pasos de $5 \mathrm{cmH}_{2} \mathrm{O}$, exploraron la respuesta a niveles crecientes de presión desde 40 hasta $60 \mathrm{cmH}_{2} \mathrm{O}$. Para evaluar si el pulmón era reclutado de modo completo, analizaron la oxigenación y la TC tras reducir la presión a los valores de inicio con una PEEP de $25 \mathrm{cmH}_{2} \mathrm{O}$, nivel suficiente para evitar el desreclutamiento. De los 26 pacientes con SDRA estudiados, la mitad precisó niveles $>40$ $\mathrm{cmH}_{2} \mathrm{O}$ para conseguir un reclutamiento completo. Si hubiesen explorado tan sólo el primer nivel de presión, habrían declarado a la mitad de los pacientes no respondedores. Al individualizar la maniobra sólo 2 pacientes no pudieron ser reclutados de modo completo y cada paciente fue reclutado a su nivel de presión óptimo evitando niveles de presión innecesarios.

\section{RECLUTAMIENTO Y PEEP}

Si el objetivo de las MR es la protección pulmonar, tan importante es conseguir una reexpansión pulmonar efectiva durante la MR como que ésta se mantenga en el tiempo mediante la selección de un nivel de PEEP durante y después de la MR capaz de mantener la estabilidad espiratoria del pulmón reexpandido. Al igual que las presiones de apertura, el nivel de PEEP necesario es variable y sólo puede determinarse tras la reexpansión pulmonar. Las poblaciones alveolares reexpandidas tienen diferentes presiones críticas de cierre, lógicamente mayores, por la localización del colapso preferentemente en las regiones dependientes del pulmón que soportan el peso del pulmón suprayacente ${ }^{9}$. En este sentido, si tras la MR se reinstau- ra sin más el nivel de PEEP previo ${ }^{11,18,28,29,34-37}$ o un nivel basado en criterios no relacionados con la presión de cierre como es el punto de inflexión inferior de la curva volumen-presión ${ }^{11,28}$, se corre el riesgo de un recolapso precoz, cuando la nueva presión de cierre sea mayor que el nivel de PEEP preajustado, con la consecuente pérdida inmediata de los beneficios fisiológicos obtenidos durante la MR. En estudios experimentales se ha mostrado que el colapso ocurre a los pocos segundos tras retirar la PEEP ${ }^{38}$. Grasso et al y Oczenski et al observaron que en pacientes con SDRA la respuesta favorable obtenida durante los primeros 2-3 min tras MR se perdía a los 20-30 min tras reinstaurar la ventilación con el nivel de PEEP previo. Ello contrasta con los efectos sostenidos conseguidos en estudios en los que se ajustó el nivel de PEEP tras la MR buscando la prevención del recolapSo $^{10,12,31}$.

Los fenómenos de reclutamiento y colapso son difíciles de monitorizar y la búsqueda nivel de PEEP que evite el colapso espiratorio no es fácil de determinar en el contexto clínico. Se ha propuesto la determinación decremental de PEEP tras la MR en la que partiendo de un nivel de PEEP elevado mantenido durante la MR, se reduce éste gradualmente hasta identificar la presión de colapso o de cierre: el descenso en la oxigenación a un valor por debajo del $10 \%$ de la $\mathrm{PaO}_{2}$ máxima alcanzada tras el reclutamiento ${ }^{10,12} \mathrm{o}$ el máximo valor de distensibilidad dinámica ${ }^{39}$. Una vez identificado el punto de colapso la PEEP, se establece definitivamente a un nivel 2 a $3 \mathrm{cmH}_{2} \mathrm{O}$ superior al punto de colapso tras una nueva MR.

\section{TOLERANCIA A LAS MANIOBRAS DE RECLUTAMIENTO}

La elevación de la presión intratorácica a los niveles necesarios para conseguir un reclutamiento efectivo puede resultar en efectos hemodinámicos adversos, una breve alteración en el intercambio gaseoso y en barotrauma. No obstante, en la mayoría de los estudios clínicos publicados las MR fueron bien toleradas desde el punto de vista hemodinámico sin evidencia de nuevo barotrauma. Grasso et al observaron descensos en el gasto cardiaco de un 20-30\% durante la aplicación una insuflación sostenida de $40 \mathrm{cmH}_{2} \mathrm{O}$ en una serie de pacientes con SDRA ${ }^{29}$. Lapinski et al, utilizando el mismo tipo de MR, describieron un descenso moderado de la presión arterial sistémica y un episodio de bradicardia ${ }^{35}$. En ambos casos estos efectos revirtieron minutos después de finalizada la MR. Brower et $\mathrm{al}^{34}$ documentaron la aparición de un nuevo barotrauma en una serie de 43 pacientes con SDRA.

Hay que destacar que la mayoría de los efectos hemodinámicos adversos se han producido durante la realización de una maniobra de insuflación sostenida. Lim et al mostraron que este tipo de MR resultaba en descensos mucho más marcados en el gasto cardíaco en comparación con MR realizadas en presión controlada en las que se mantuvo un ciclado inspiratorioespiratorio normal ${ }^{40}$. Es por ello que la insuflación 
sostenida como MR debería ser abandonada en favor de las maniobras de presión controlada.

\section{CONCLUSIONES}

Las MR utilizadas de forma juiciosa y protocolizada en el contexto de una estrategia de protección pulmonar pueden tener un papel importante en el manejo ventilatorio del SDRA. Deben realizarse de modo precoz e individualizado, buscando como objetivo la máxima reexpansión pulmonar posible y su mantenimiento en el tiempo, la llamada estrategia de pulmón abierto $^{6}$. El ajuste de la PEEP que evite el colapso tras la MR es indispensable. Sin él el reclutamiento no tiene justificación, ya que sólo conseguiría beneficios transitorios de la oxigenación a modo de «suspiros» que para mantenerse en el tiempo obligarían a una repetición frecuente y desaconsejable de las MR. La información acumulada en los estudios clínicos y experimentales de los últimos años ha supuesto un gran avance en el conocimiento acerca de los mecanismos, tolerancia, efectividad y utilidad de las MR aportando una visión más clara de cuál puede ser su papel en la protección pulmonar. Gracias a ello hoy nos encontramos algo más cerca de poder determinar si, como cree el autor, las MR tienen un papel relevante en la protección pulmonar y, por ende, pueden mejorar el pronóstico de los pacientes con SDRA.

\section{BIBLIOGRAFÍA}

1. Fan E, Needham DM, Stewart TE. Ventilatory management of acute lung injury and acute respiratory distress syndrome. JAMA. 2005;294:2889-96.

2. Dreyfuss D, Saumon G. Ventilator-induced lung injury: lessons from experimental studies. Am J Respir Crit Care Med. 1998; 157:294-323.

3. Amato MBP, Barbas CSV, Medeiros DM, et al. Effect of a protective-ventilation strategy on mortality in the acute respiratory distress syndrome. N Engl J Med. 1998;338:347-54.

4. The Acute Respiratory Distress Syndrome N. Ventilation with lower tidal volumes as compared with traditional tidal volumes for acute lung injury and the acute respiratory distress syndrome. $\mathrm{N}$ Engl J Med. 2000;342:1301-8.

5. Villar J, Kacmarek RM, Perez-Mendez L, Aguirre-Jaime A. A high positive end-expiratory pressure, low tidal volume ventilatory strategy improves outcome in persistent acute respiratory distress syndrome: a randomized, controlled trial. Crit Care Med. 2006;34:1311-8.

6. Lachmann B. Open up the lung and keep the lung open. Intensive Care Med. 1992;18:319-21.

7. Moran I, Zavala E, Fernandez R, et al. Recruitment manoeuvres in acute lung injury/acute respiratory distress syndrome. Eur Respir J Suppl. 2003;42:S37-42.

8. Gattinoni L, Caironi P, Pelosi P, Goodman LR. What has computed tomography taught us about the acute respiratory distress syndrome? Am J Respir Crit Care Med. 2001;164:1701-11.

9. Gattinoni L, Pelosi P, Crotti S, Valenza F. Effects of positive end-expiratory pressure on regional distribution of tidal volume and recruitment in adult respiratory distress syndrome. Am J Respir Crit Care Med. 1995;151:1807-14.

10. Borges JB, Okamoto VN, Matos GFJ, et al. Reversibility of lung collapse and hypoxemia in early acute respiratory distress syndrome. Am J Respir Crit Care Med. 2006;174:268-78.

11. Bugedo G, Bruhn A, Hernandez G, et al. Lung computed tomography during a lung recruitment maneuver in patients with acute lung injury. Intensive Care Med. 2003;29:218-25.
12. Schreiter D, Reske A, Stichert B, et al. Alveolar recruitment in combination with sufficient positive end-expiratory pressure increases oxygenation and lung aeration in patients with severe chest trauma. Crit Care Med. 2004;32:968-75.

13. Gattinoni L, Caironi $P$, Cressoni M, et al. Lung recruitment in patients with the acute respiratory distress syndrome. N Engl J Med. 2006;354:1775-86.

14. Muscedere JG, Mullen JBM, Gan K, et al. Tidal ventilation at low airway pressures can augment lung injury. Am J Respir Crit Care Med. 1994;149:1327-34.

15. Chiumello D, Pristine G, Slutsky AS. Mechanical ventilation affects local and systemic cytokines in an animal model of acute respiratory distress syndrome. Am J Respir Crit Care Med. 1999;160:109-16.

16. Rimensberger $\mathrm{P}$, Cox $\mathrm{P}$, Frondova $\mathrm{H}$, et al. The open lung during small tidal volume ventilation: Concepts of recruitment and «optimal» positive end-expiratory pressure. Crit Care Med. 1999;27:1946-52.

17. Cereda M, Foti G, Musch G, et al. Positive end-expiratory pressure prevents the loss of respiratory compliance during low tidal volume ventilation in acute lung injury patients. Chest. 1996;109:480-5.

18. Johannigman JA, Miller SL, Davis BR, et al. Influence of low tidal volumes on gas exchange in acute respiratory distress syndrome and the role of recruitment maneuvers. J Trauma. 2003;54:320-5.

19. Richard J-C, Maggiore SM, Jonson B, et al. Influence of tidal volume on alveolar recruitment. Respective role of PEEP and a recruitment maneuver. Am J Respir Crit Care Med. 2001;163:1609-13.

20. Grasso S, Stripoli T, De Michele M, et al. ARDSnet Ventilatory Protocol and alveolar hyperinflation: role of positive end-expiratory pressure. Am J Respir Crit Care Med. 2007; 176:761-7.

21. Terragni PP, Rosboch G, Tealdi A, et al. Tidal hyperinflation during low tidal volume ventilation in acute respiratory distress syndrome. Am J Respir Crit Care Med. 2007;175:160-6.

22. Brower RG, Lanken PN, MacIntyre N, et al. Higher versus lower positive end-expiratory pressures in patients with the acute respiratory distress syndrome. N Engl J Med. 2004;351:327-36.

23. Meade MO, Cook DJ, Guyatt GH, et al. Ventilation strategy using low tidal volumes, recruitment maneuvers, and high positive end-expiratory pressure for acute lung injury and acute respiratory distress syndrome: a randomized controlled trial. JAMA. 2008;299:637-45.

24. Mercat A, Richard J-CM, Vielle B, et al. Positive end-expiratory pressure setting in adults with acute lung injury and acute respiratory distress syndrome: a randomized controlled trial. JAMA. 2008;299:646-55.

25. Grasso S, Fanelli V, Cafarelli A, et al. Effects of high versus low positive end-expiratory pressures in acute respiratory distress syndrome. Am J Respir Crit Care Med. 2005;171:1002-8.

26. Richard JC, Maggiore S, Mercat A. Where are we with recruitment maneuvers in patients with acute lung injury and acute respiratory distress syndrome? Curr Opin Crit Care. 2003;9:22-7.

27. Richard JC, Maggiore SM, Mercat A. Clinical review: Bedside assessment of alveolar recruitment. Crit Care. 2004;8: 163-9.

28. Villagra ANA, Ochagavia ANA, Vatua S, et al. Recruitment maneuvers during lung protective ventilation in acute respiratory distress syndrome. Am J Respir Crit Care Med. 2002;165:165-70.

29. Grasso S, Mascia L, Del Turco M, et al. Effects of recruiting maneuvers in patients with acute respiratory distress syndrome ventilated with protective ventilatory strategy. Anesthesiology. 2002;96:795-802.

30. Povoa P, Almeida E, Fernandes A, et al. Evaluation of a recruitment maneuver with positive inspiratory pressure and high PEEP in patients with severe ARDS. Acta Anaesthesiol Scand. 2004;48:287-93.

31. Tugrul S, Akinci O, Ozcan PE, et al. Effects of sustained inflation and postinflation positive end-expiratory pressure in acute respiratory distress syndrome: focusing on pulmonary and extrapulmonary forms. Crit Care Med. 2003;31:738-44.

32. Rothen HU, Sporre B, Engberg G, et al. Re-expansion of atelectasis during general anaesthesia: a computed tomography study. Br J Anaesth. 1993;71:788-95. 
33. Bein T, Reber A, Metz C, et al. Acute effects of continuous rotational therapy on ventilation-perfusion inequality in lung injury. Intensive Care Med. 1998;24:132-7.

34. Brower RG, Morris A, MacIntyre N, et al. Effects of recruitment maneuvers in patients with acute lung injury and acute respiratory distress syndrome ventilated with high positive endexpiratory pressure. Crit Care Med. 2003;31:2592-7.

35. Lapinsky SE, Aubin M, Mehta S, et al. Safety and efficacy of a sustained inflation for alveolar recruitment in adults with respiratory failure. Intensive Care Med. 1999;25:1297-301.

36. Lim CM, Jung H, Koh Y, et al. Effect of alveolar recruitment maneuver in early acute respiratory distress syndrome according to antiderecruitment strategy, etiological category of diffuse lung injury, and body position of the patient. Crit Care Med. 2003;31: 411-8.
37. Oczenski W, Hormann C, Keller C, et al. Recruitment maneuvers after a positive end-expiratory pressure trial do not induce sustained effects in early adult respiratory distress syndrome. Anesthesiology. 2004;101:620-5.

38. Neumann P, Berglund JE, Fernandez Mondejar E, et al. Dynamics of lung collapse and recruitment during prolonged breathing in porcine lung injury. J Appl Physiol. 1998;85: 1533-43.

39. Suarez-Sipmann F, Böhm S, Tusman G, et al. Use of dynamic compliance for open lung positive end-expiratory pressure titration in an experimental study. Crit Care Med. 2007;35: 214-21.

40. Lim SC, Adams AB, Simonson DA, et al. Transient hemodynamic effects of recruitment maneuvers in three experimental models of acute lung injury. Crit Care Med. 2004;32:2378-84. 